\title{
Tunnel Intergrowth Structures in Manganese Dioxide and Their Influence on Ion Storage
}

Yifei Yuan ${ }^{1,2}$, Bryan Byles ${ }^{3}$, Wentao Yao ${ }^{1}$, Kun $\mathrm{He}^{1}$, Meng Cheng ${ }^{1}$, Jun $\mathrm{Lu}^{2}$, Khalil Amine ${ }^{2}$, Ekaterina Pomerantseva $^{3}$, Reza Shahbazian-Yassar ${ }^{1}$

1. Department of Mechanical and Industrial Engineering, University of Illinois at Chicago, Chicago, IL 60607, USA

2. Chemical Science and Engineering Division, Argonne National Laboratory, 9700 S. Cass Avenue, Argonne, Illinois 60439, USA

3. Department of Materials Science and Engineering, Drexel University, Philadelphia, PA 19104, USA

Controlled synthesis of homogeneous tunnel-structured materials has been a grand challenge due to the lack of understanding on heterogeneities associated with tunnel structuring and abnormalities. The presence of tunnel structure heterogeneity disables the quantification and optimization of the performance of various tunnel-structured functional materials in their targeted applications such as catalysis, water purification, energy storage, and sensing [1]. Manganese dioxide $\left(\mathrm{MnO}_{2}\right)$ is a highly polymorphic material with several well known tunnel-structured phases such as $\beta-\mathrm{MnO}_{2}, \alpha-\mathrm{MnO}_{2}$, and $\mathrm{T}-\mathrm{MnO}_{2}$ featuring $1 \times 1$ tunnels, $2 \times 2$ tunnels, and $3 \times 3$ tunnels, respectively [2]. For over a century, the tunnel-dependent properties of $\mathrm{MnO}_{2}$ in areas such as catalysis and ion transport have been gradually realized, and numerous studies have been devoted to the synthesis of homogeneous tunnel phases [3]. Until recently, the structural details of the tunnel phases have not been revealed clearly, especially down to the atomic level [4].

Here, we demonstrate for the first time the correlation between homogeneity of the tunnels and their dimensions. Utilizing state-of-the-art aberration-corrected scanning transmission electron microscopy that is sensitive to local structure information, we found that while $\beta-\mathrm{MnO}_{2}$ possesses a homogeneous $1 \times 1$ tunnel phase, the tunnel homogeneity decreases when tunnel dimension increasing from $2 \times 2$ tunnelstructured $\alpha-\mathrm{MnO}_{2}$ to $3 \times 3$ tunnel-structured $\mathrm{T}-\mathrm{MnO}_{2}$. For $\alpha-\mathrm{MnO}_{2}$, a complicated $2 \times 3$ and $2 \times 4$ tunnel intergrowth as well as tunnel shift $(\sim 7 \AA)$ and rotation $\left(\sim 90^{\circ}\right)$ with smaller $1 \times 2$ tunnels accommodating the structure mismatch are disclosed. For $\mathrm{T}-\mathrm{MnO}_{2}$, a larger density of defective tunnel intergrowths is observed. The existence of large tunnels ( $>10 \AA$ ) such as $3 \times 4,3 \times 5,3 \times 6,4 \times 4,4 \times 6,5 \times 3,5 \times 4$, and $5 \times 5$ tunnels is discovered in this work for the first time. Such tunnels are found to be stable within the $3 \times 3$ tunnel-dominated $\mathrm{T}-\mathrm{MnO}_{2}$ matrix. The complicated tunnel intergrowths discovered in this work are shown to affect the ion storage behavior in the tunnel-structured materials as revealed by in situ TEM of a single $\mathrm{MnO}_{2}$ nanowire exhibiting such tunnel heterogeneity. The findings of this work call for renewed attention to the synthesis of homogeneous tunnel-specific $\mathrm{MnO}_{2}$ phases for applications where tunnel homogeneity is desired. 


\section{References}

[1]. N Zhang, et al., Nat. Commun. 8 (2017), 405.

[2]. D Kitchaev, et al., J. Am. Chem. Soc. 139 (2017), p. 2672.

[3]. S Turner and P. Buseck, Science 203 (1979), p. 456.

[4]. Y Yuan, et al., ACS Nano 10 (2016), p. 539. 